\title{
Unprecedented Treatment Strategy of Aquatic Environments. Oxidative Degradation of Penicillin G by Chromium Trioxide in Acidic Media and the Impact of Metal Ion Catalysts: Kinetics and Mechanistic Insights
}

\author{
Ahmed Fawzy 1,2,*, Arafat Toghan ${ }^{3,4}$ \\ ${ }^{1}$ Chemistry Department, Faculty of Applied Sciences, Umm Al-Qura University, Makkah 21955, Saudi Arabia \\ ${ }^{2}$ Chemistry Department, Faculty of Science, Assiut University, Assiut 71516, Egypt \\ ${ }^{3}$ Chemistry Department, Faculty of Science, South Valley University, Qena, 83523, Egypt \\ ${ }^{4}$ Chemistry Department, College of Science, Imam Mohammad Ibn Saud Islamic University (IMSIU), Riyadh \\ 11623, Saudi Arabia
}

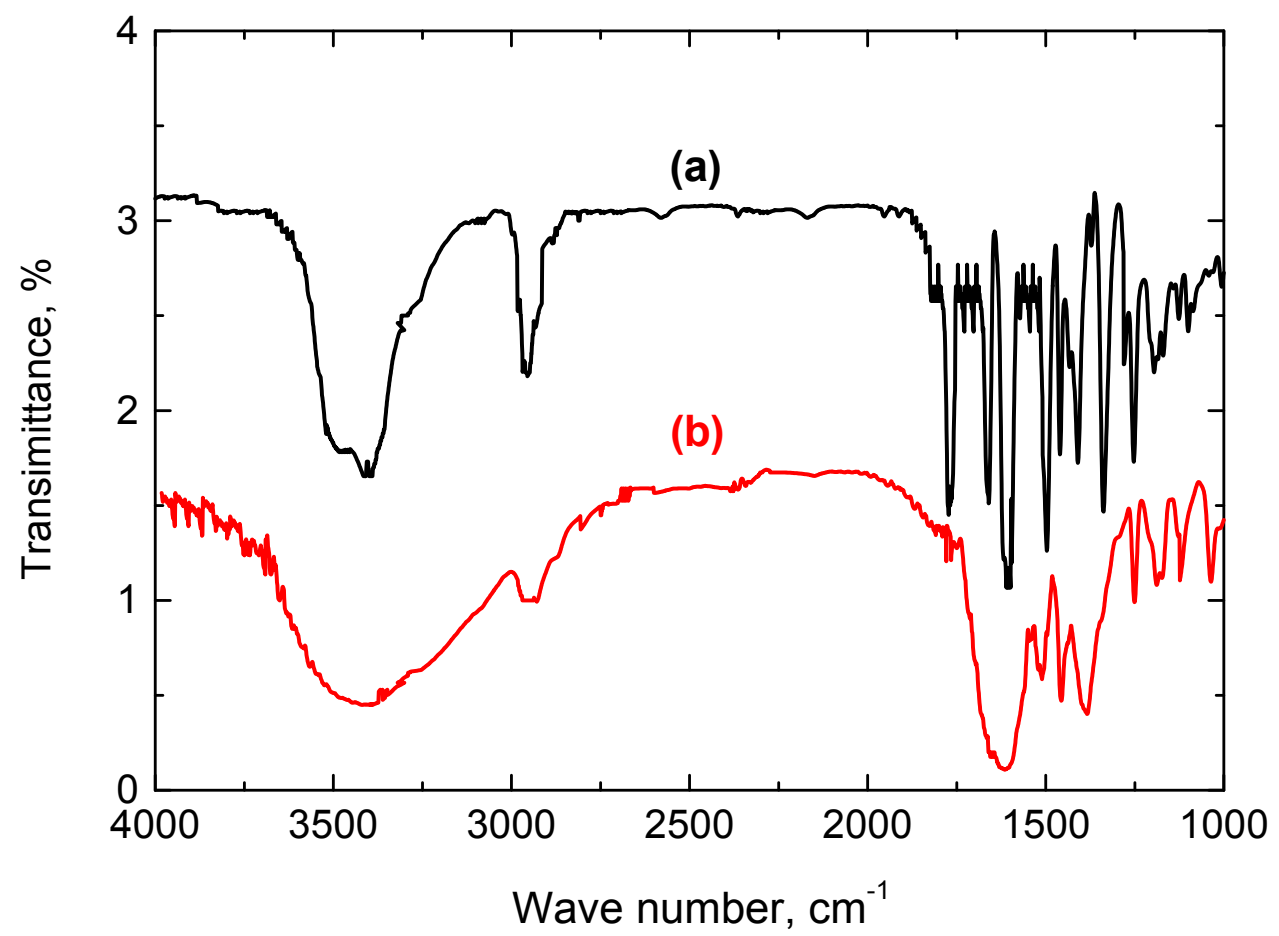

Figure S1. FT-IR spectra of: (a) penicillin G and, (b) its oxidation products as a results of its reaction with chromium trioxide oxidant in perchloric acid medium. 


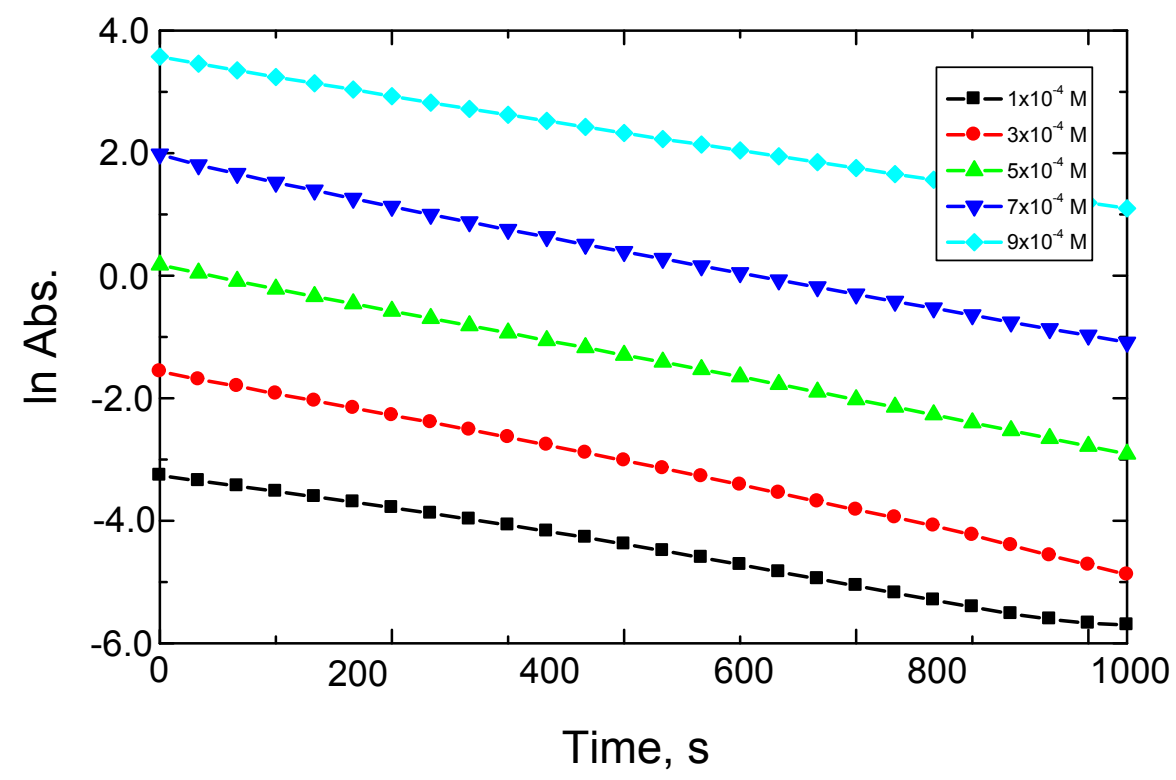

Figure S2. Graphs of $\ln$ Abs. versus time in the oxidation of penicillin $\mathrm{G}$ by chromium trioxide in sulfuric acid solution. $[\mathrm{Pen}]=2.0 \times 10^{-2},\left[\mathrm{H}^{+}\right]=1.0$ and $I=2.0 \mathrm{~mol} \mathrm{dm}^{-3}$ at $298 \mathrm{~K}$.

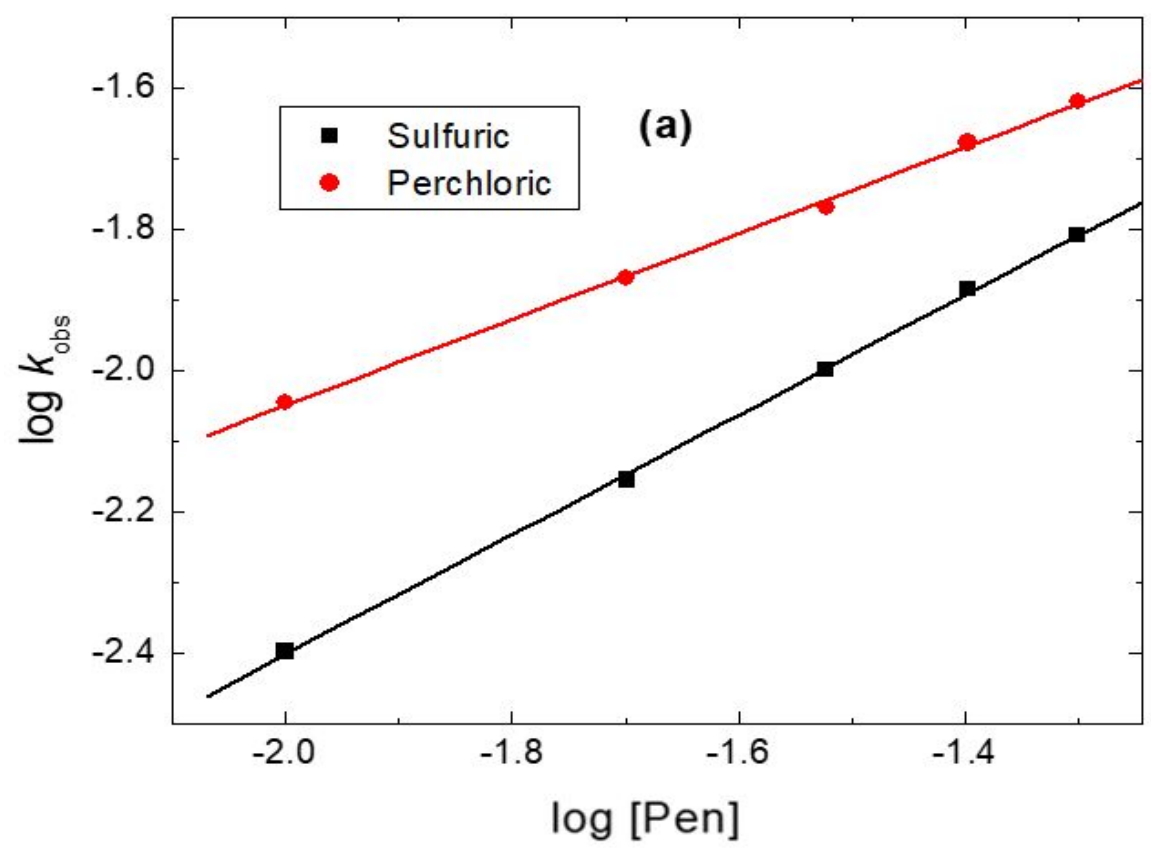

Figure S3. $\log k_{\text {obs }}$ versus $\log$ [Pen] graphs in the oxidation of penicillin $\mathrm{G}$ by chromium trioxide in sulfuric and perchloric acid solutions. $\left[\mathrm{Cr}^{\mathrm{VI}}\right]=5.0 \times 10^{-4},\left[\mathrm{H}^{+}\right]=1.0$ and $I=2.0 \mathrm{~mol} \mathrm{dm}^{-3}$ at $298 \mathrm{~K}$. 


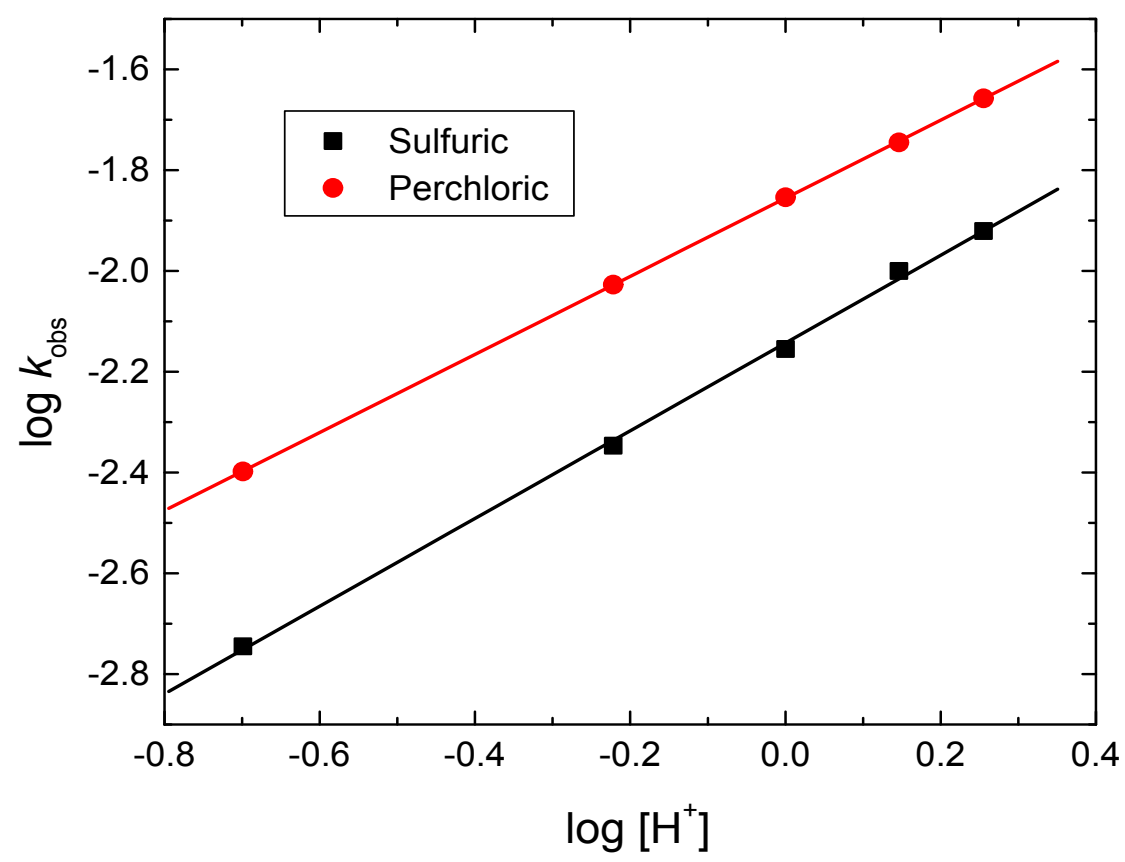

Figure S4. $\log k_{\mathrm{obs}}$ versus $\log \left[\mathrm{H}^{+}\right]$graphs in the oxidation of penicillin $\mathrm{G}$ by chromium trioxide in sulfuric and perchloric acid solutions. $[\mathrm{Pen}]=3.0 \times 10^{-2},\left[\mathrm{Cr}^{\mathrm{VI}}\right]=5.0 \times 10^{-4}$ and $I=2.0 \mathrm{~mol}$ $\mathrm{dm}^{-3}$ at $298 \mathrm{~K}$. 